International Journal of Biomathematics

Vol. 8, No. 6 (2015) 1599001 (8 pages)

(C) World Scientific Publishing Company

DOI: $10.1142 / \mathrm{S} 1793524515990016$

\title{
Author index (Volume 8)
}

Abdelrazec, A., see

Mastroberardino, A.

Abolpour, N., see

Golpaygani, A. T.

Adlakha, N., see Jha, A.

Adlakha, N., see

Makrariya, A.

Ahmad, B., see Hayat,

$\mathrm{T}$.

Ahmad, B., see Hayat, T.

Ahmad, B., see

Nyamoradi, N.

Akbar, N. S. \& Khan,

Z. H., Heat transfer analysis of bi-viscous ciliary motion fluid

Akbar, N. S. \& Nadeem, S., Mathematical analysis of PhanThien-Tanner fluid model for blood in arteries

Akbar, N. S., see Maraj,

E. N.

Akbar, N. S.,

Biomathematical

analysis of carbon

nanotubes due to

ciliary motion

Akbar, N. S., Mixed convection analysis for blood flow through arteries on Williamson fluid model

Akbar, N. S.,

Biomathematical study of Sutterby fluid model for blood flow in stenosed arteries

Al-Neama, M. W., Reda, N. M. \& Ghaleb, F. F. M., Fast vectorized distance matrix
5 (2015) 1550064

4 (2015) 1550045

\section{4 (2015) 1550047 \\ 6 (2015) 1550078 \\ multiple sequence \\ alignment on \\ multi-cores}

1 (2015) 1550002

6 (2015) 1550074

5 (2015) 1550061

6 (2015) 1550073

6 (2015) 1550076

2 (2015) 1550026

1 (2015) 1550005

2 (2015) 1550023

6 (2015) 1550075

Elaiw, A. M. T. instability in a diffusive SIS Kordrostami, S. A. A. R.

Awgichew, G., see

Vajravelu, K.

Babujee, J. B. \&

Wiener index of graphs M. A.

Bai, M., see Xu, S.

Bajelani, K., see Golpaygani, A. T. Sivakumar, M. see Prakash, M.

Bhatia, S. K., see Chauhan, S. computation for

AlShamrani, N. H., see

6 (2015) 1550084

Alsaadi, F., see Hayat,

5 (2015) 1550058

2 (2015) 1550024

Aly, S., Khenous, H. B. \& Hussien, F., Turing epidemiological model

Amirteimoori, A., see

Arshad, S., see Sohail,

Asl, M. S., see Haghighi,

Senbagamalar, J., On

Wiener and terminal

Badshah, Q., see Khan,

Balachandran, K., see

Balasubramaniam, P.,

Bekir, A., see Güner, Ö.

Borsali, F. \& Yadi, K., Contribution to the study of the effect of the interspecificity on a two nutrients competition model
5 (2015) 1550066

1 (2015) 1550006

3 (2015) 1550034

5 (2015) 1550069

4 (2015) 1550056

4 (2015) 1550055

6 (2015) 1550082

2 (2015) 1550018

6 (2015) 1550078

1 (2015) 1550013

5 (2015) 1550059

1 (2015) 1550003

6 (2015) 1550083

1 (2015) 1550008 
Buonomo, B., Analysis of a malaria model with mosquito host choice and bed-net control

Chattopadhyay, J., see Das, K. P.

6 (2015) 1550077

2 (2015) 1550015

Chauhan, S., Bhatia, S. K. \& Gupta, S., Effect of pollution on dynamics of SIR model with treatment

6 (2015) 1550083

Chen, F., see Chen, L.

Chen, L. \& Chen, F., Extinction in a discrete Lotka-Volterra competitive system with the effect of toxic substances and feedback controls

Chen, L., see Ji, X.

Chen, S., see Wu, Q.

Chen, Y. P., see Tan, R. H.

Chen, Y., An island biogeography model for beta diversity and endemism: The roles of speciation, extinction and dispersal

Chen, Y.-Z., see Zhou, L.-Y.

Cheng, Y., see Mastroberardino, A.

Das, K. P. \&

Chattopadhyay, J., A mathematical study of a predator-prey model with disease circulating in the both populations

Das, K. P., Alternative food and external source of infection stabilize predator-prey oscillations - A conclusion drawn from an eco-epidemiological model

De, A., Maity, K. \& Maiti, M., Stability analysis of combined project of fish, broiler and ducks: Dynamical

1 (2015) 1550012

5 (2015) 1550062

2 (2015) 1550015

3 (2015) 1550032 system in imprecise environment 5 (2015) 1550067

Doyle, D. J., see Golpaygani, A. T.

6 (2015) 1550078

4 (2015) 1550043

Du, Y., see Qu, J. Y.

Elaiw, A. M. \&

AlShamrani, N. H.,

Global properties of nonlinear humoral immunity viral infection models 5 (2015) 1550058

Ellahi, R., see Riaz, A.

6 (2015) 1550081

Falconi, M., see Sáez, E.

Farooq, A. A., see

Siddiqui, A. M.

Feng, C. \& Huang, J., Almost periodic solutions of

nonautonomous

Lotka-Volterra

competitive systems with dominated delays

Ferrari, M. A., see Pérez, M. C.

2 (2015) 1550022

2 (2015) 1550016

2 (2015) 1550019

4 (2015) 1550049

Freidoonimehr, N.,

Rostami, B. \& Rashidi, M. M., Predictor homotopy analysis method for nanofluid flow through expanding or contracting gaps with permeable walls

4 (2015) 1550050

Gagoshidze, M., see Jangveladze, T.

Gao, H., see Li, M.

Gao, S., see Xie, D.

Ghaffari, A., see Nazari, M.

5 (2015) 1550057

6 (2015) 1550072

2 (2015) 1550021

3 (2015) 1550036

Ghaleb, F. F. M., see Al-Neama, M. W.

Golpaygani, A. T., Abolpour, N., Hassani, K., Bajelani, K. \& Doyle, D. J., Detection and identification of $\mathrm{S} 1$ and S2 heart sounds using wavelet decomposition method

Gong, Y., see Li, M.

González-Olivares, E., see Sáez, E.

Güner, Ö. \& Bekir, A., Exact solutions of some fractional
6 (2015) 1550084

6 (2015) 1550078

6 (2015) 1550072

$2(2015) 1550022$ 
differential equations arising in mathematical biology

1 (2015) 1550003

Gupta, S., see Chauhan, S.

Haghighi, A. R. \& Asl, M. S., Mathematical modeling of micropolar fluid flow through an overlapping arterial stenosis

Hassani, K., see Golpaygani, A. T.

Hawkins, J. \& Molinek, automata models for chronic disease progression

Hayat, T., Rafiq, M. \& Ahmad, B., Combined effects of rotation and thermal radiation on peristaltic transport of Jeffrey fluid

Hayat, T., Rafiq, M., Ahmad, B. \& Yasmin, H., Effect of melting heat transfer on peristalsis in the presence of thermal radiation and Joule heating Yasmin, H. \& Alsaadi, F., Simultaneous effects of Hall current and thermal deposition in peristaltic transport of Eyring-Powell fluid

Huang, J., see Feng, C.

Hussien, F., see Aly, S.

Islam, S., see Khan, M. A.

Jabłońska-Sabuka, M., see Nannyonga, B.

Jain, N., see Prakash, O.

Jangveladze, T.,

Kiguradze, Z.,

Gagoshidze, M. \&

Nikolishvili, M.,

Stability and

convergence of the

variable directions

difference scheme for

one nonlinear

6 (2015) 1550083

4 (2015) 1550056

6 (2015) 1550078

6 (2015) 1550085

6 (2015) 1550073

2 (2015) 1550019

6 (2015) 1550082

5 (2015) 1550065

1 (2015) 1550010 D., Markov cellular

Hayat, T., Tanveer, A., two-dimensional

model

5 (2015) 1550057

Javed, S., see Sohail, A.

Javidi, M. \& Nyamoradi, N., Dynamic analysis of a fractional order system

5 (2015) 1550069

Javidi, M., see

Nyamoradi, N.

Jha, A. \& Adlakha, N.,

Two-dimensional finite element model to study unsteady state $\mathrm{Ca}^{2+}$ diffusion in neuron involving ER LEAK and $S E R C A$

6 (2015) 1550079

6 (2015) 1550076

1 (2015) 1550002

Ji, X., Yuan, S. \& Chen, L., A pest control model with

state-dependent impulses

Jia, J. W. \& Lv, T. T., Study of two-nutrient and two-microorganism chemostat model with pulsed input in a polluted environment

Jiang, X., see Yang, Y.

Jiang, X.-C., see Zhou, L.-Y.

Kamal, M. S., see Nimmy, S. F.

Khan, M. A., Khan, Y., Badshah, Q. \& Islam, S., Global stability of SEIVR epidemic model with generalized incidence and preventive vaccination

6 (2015) 1550082

1 (2015) 1550006

Khan, Y., see Khan, M. A.

Khan, Z. H., see Akbar,

Khenous, H. B., see Aly,

4 (2015) 1550042

2 (2015) 1550020

5 (2015) 1550070

5 (2015) 1530001 N. S. $\mathrm{S}$.

2 (2015) 1550026

1 (2015) 1550006

Kiguradze, Z., see Jangveladze, $\mathrm{T}$.

Kong, X., see Ou Yang, H.-B.

5 (2015) 1550057

3 (2015) 1550037
Kordrostami, S., Amirteimoori, A. \& Noveiri, M. J. S., Restricted variation in data envelopment 
analysis with

undesirable factors in nature

3 (2015) 1550034

Kothandapani, M.,

Prakash, J. \& Srinivas,

S., Peristaltic

transport of a MHD

Carreau fluid in a

tapered asymmetric

channel with perme-

able walls

4 (2015) 1550054

Kumar, A., see Yadav,

A.

Kumar, D., see Prakash, O.

Kuniya, T., see Muroya, Y.

Lahrouz, A., Dynamics of a delayed epidemic model with varying immunity period and nonlinear transmission

Li, M., Gao, H., Sun, C. \& Gong, Y., Analysis of a mutualism model with stochastic

perturbations

Li, S., see Ou Yang, H.-B.

Li, S., see Tan, C.

Liu, H., see Mastroberardino, A.

Liu, Z. J., see Tan, R. H.

Luboobi, L. S., see Nannyonga, B.

Luo, D., see Lv, G.

Lv, G. \& Luo, D., Entire solutions for some reaction-diffusion systems

Lv, T. T., see Jia, J. W.

Maiti, M., see De, A.

Maity, K., see De, A.

Makinde, O. D., see

Prakash, O.

Makrariya, A. \&

Adlakha, N.,

Two-dimensional finite element model to study temperature distribution in peripheral regions of extended spherical human organs

1 (2015) 1550001

1 (2015) 1550010

4 (2015) 1550048

2 (2015) 1550027

6 (2015) 1550072

3 (2015) 1550037

1 (2015) 1550004

4 (2015) 1550047

5 (2015) 1550062

5 (2015) 1550065

4 (2015) 1550052

4 (2015) 1550052

4 (2015) 1550042

5 (2015) 1550067

5 (2015) 1550067

1 (2015) 1550010 involving uniformly

perfused tumors

6 (2015) 1550074

Maqbool, K., see Sohail, A.

5 (2015) 1550069

Maraj, E. N., Akbar, N. S. \& Nadeem, S., Mathematical study for peristaltic flow of Williamson fluid in a curved channel

1 (2015) 1550005
Mastroberardino, A., Cheng, Y., Abdelrazec,

A. \& Liu, H., Mathematical modeling of the HIV/AIDS epidemic in Cuba

Matadi, M. B., Singularity and Lie group analyses for tuberculosis with exogenous reinfection

Misra, O. P., Sinha, P. \& Singh, C., Dynamics of one-prey two-predator system with square root functional response and time lag

3 (2015) 1550029

Molinek, D., see

Hawkins, J.

6 (2015) 1550085

Muroya, Y. \& Kuniya, T., Global stability for a delayed multi-group SIRS epidemic model with cure rate and incomplete recovery rate

4 (2015) 1550048

Murugesu, R., see Rajasingh, J.

Nadeem, S., see Akbar, N. S.

3 (2015) 1550033

5 (2015) 1550064

Nadeem, S., see Maraj, E. N.

1 (2015) 1550005

6 (2015) 1550081

Nadeem, S., see Riaz, A.

Nannyonga, B., Luboobi, L. S., Tushemerirwe, P. \& Jabłońska-Sabuka, M., Using contaminated tools fuels outbreaks of Banana Xanthomonas wilt: $A n$ optimal control study within plantations using RungeKutta fourth-order algorithms

5 (2015) 1550065 
Nasha, V., see Sharma, M. K.

3 (2015) 1550028

Nazari, M. \& Ghaffari, A., The effect of finite duration inputs on the dynamics of a system: Proposing a new approach for cancer treatment

3 (2015) 1550036

Nikolishvili, M., see Jangveladze, $\mathrm{T}$.

Nimmy, S. F. \& Kamal, M. S., Next generation sequencing under de novo genome assembly

Noveiri, M. J. S., see Kordrostami, S.

Nyamoradi, N., Javidi, M. \& Ahmad, B., Dynamics of SVEIS epidemic model with distinct incidence

Nyamoradi, N., see Javidi, M.

Obaid, H. A., Ouifki, R. \& Patidar, K. C., Analysis of an HIV model with distributed delay and behavior change

Osuna, O. \&

Vargas-De-León, C., Construction of Dulac functions for mathematical models in population biology

Ou Yang, H.-B., Li, S., Zhang, P. \& Kong, X., Model penicillin fermentation by least squares support vector machine with tuning based on amended harmony search

Ouifki, R., see Obaid, H. A.

Pan, S., Asymptotic speed of spreading of a nonlocal delayed equation without quasimonotonicity

Patidar, K. C., see Obaid, H. A.

Peng, H., see Wang, L.
4 (2015) 1550046

2 (2015) 1550017

5 (2015) 1550057

5 (2015) 1530001

3 (2015) 1550034

6 (2015) 1550076

6 (2015) 1550079

2 (2015) 1550017

3 (2015) 1550035

3 (2015) 1550037

2 (2015) 1550017

5 (2015) 1550063
Pérez, M. C., Saavedra, E. A. \& Ferrari, M. A., Dynamics of populations with two sexes

4 (2015) 1550049

Prakash, J., see

Kothandapani, M.

Prakash, M. \& Balasubramaniam, P., Stability and multiparametric Hopf bifurcation analyses of viral infection model with time delay

4 (2015) 1550054

5 (2015) 1550059

Prakash, O., Makinde, O. D., Singh, S. P., Jain, N. \& Kumar, D., Effects of stenoses on non-Newtonian flow of blood in blood vessels

1 (2015) 1550010

Qiu, Y., see Wang, L.

Qu, J. Y., Wang, R. B. \& Du, Y., Measuring effects of different noises in a model using ISI-distance methods

5 (2015) 1550063

4 (2015) 1550043

Radhakrishnamacharya, G., see Vajravelu, K.

Rafiq, M., see Hayat, T. Rafiq, M., see Hayat, T.

Rajasingh, J., Murugesu, R. \& Syed Shabudeen, P., A reactiondiffusion model of forest boundary with seed dynamics

Rana, M. A., see Siddiqui, A. M.

Rashidi, M. M., see Freidoonimehr, N.

Reda, N. M., see Al-Neama, M. W.

Riaz, A., Nadeem, S. \& Ellahi, R., Effects of the wall properties on unsteady peristaltic flow of an EyringPowell fluid in a three-dimensional rectangular duct

Rostami, B., see Freidoonimehr, N. Rupšys, P., Generalized fixed-effects and
4 (2015) 1550055

5 (2015) 1550061

6 (2015) 1550073

3 (2015) 1550033

2 (2015) 1550016

4 (2015) 1550050

6 (2015) 1550084

6 (2015) 1550081

4 (2015) 1550050 
mixed-effects para-

meters height-

diameter models with

diffusion processes

Saavedra, E. A., see Pérez, M. C.

Sáez, E., Stange, E.,

Szántó, I.,

González-Olivares, E.

\& Falconi, M., Chaotic

dynamics and

coexistence in a three

species interaction

model

Samanta, G. P., see

Sharma, S.

Sambath, M., see

Sivakumar, M.

Senbagamalar, J., see Babujee, J. B.

Sharma, M. K., Sharma, P. R. \& Nasha, V., Pulsatile blood flow through stenosed artery with axial translation

Sharma, P. R., see Sharma, M. K.

Sharma, S. \& Samanta, G. P., Stability analysis and optimal control of an epidemic model with vaccination

Shit, G. C., see Sinha, A.

Siddiqui, A. M., Farooq, A. A. \& Rana, M. A., An investigation of non-Newtonian fluid flow due to metachronal beating of cilia in a tube

Singh, C., see Misra, O. P.

Singh, S. P., see Prakash, O.

Sinha, A. \& Shit, G. C., Oscillatory blood flow through a capillary in presence of thermal radiation

Sinha, P., see Misra,

O. P.

Sivakumar, M., Sambath, M. \& Balachandran,
5 (2015) 1550060

4 (2015) 1550049

2 (2015) 1550022

3 (2015) 1550030

1 (2015) 1550013

5 (2015) 1550066

3 (2015) 1550028

3 (2015) 1550028

3 (2015) 1550030

1 (2015) 1550014

2 (2015) 1550016

3 (2015) 1550029

1 (2015) 1550010

1 (2015) 1550014

3 (2015) 1550029
K., Stability and Hopf bifurcation analysis of a diffusive predatorprey model with Smith growth

1 (2015) 1550013

Sohail, A., Arshad, S., Javed, S. \& Maqbool, K., Numerical analysis of fractional-order tumor model

5 (2015) 1550069

Srinivas, S., see

Kothandapani, M.

Srivastava, P. K., see Yadav, A.

4 (2015) 1550054

1 (2015) 1550001

2 (2015) 1550022

6 (2015) 1550072

1 (2015) 1550007

Sun, J., see Zhang, J.

Sun, X. \& Wei, J., Dynamics of an infection model with two delays

Syed Shabudeen, P., see

Rajasingh, J.

5 (2015) 1550068

3 (2015) 1550033

2 (2015) 1550022

Tan, C., Li, S. \& Zhu, P., 4D Graphical representation research of DNA sequences

1 (2015) 1550004

Tan, R. H., Xiang, H. L., Chen, Y. P. \& Liu, Z. J., Dynamics behaviors of a delayed competitive system in a random environment

Taneja, H. C., see Thapliyal, R.

Tanveer, A., see Hayat, T.

Thapliyal, R. \& Taneja, H. C., On Rényi entropies of order statistics

5 (2015) 1550062

6 (2015) 1550080

2 (2015) 1550024

Tian, B., Yang, L. \& Zhong, S., Global stability of a stochastic predator-prey model with Allee effect

Tushemerirwe, P., see Nannyonga, B.

Vajravelu, K., Awgichew, G. \& Radhakrishnamacharya, G., The effects of slip condition and multiple stenoses on micropolar fluid
6 (2015) 1550080

4 (2015) 1550044

5 (2015) 1550065 
flow through a channel of non-uniform

cross-section

4 (2015) 1550055

Vargas-De-León, C., see Osuna, O.

Vargas-De-León, C., Global stability for multi-species

Lotka-Volterra cooperative systems: One hyper-connected mutualistic-species

Wang, L., Peng, H., Zheng, J. \& Qiu, Y., A new 2D graphical representation of protein sequence and its application

Wang, L., Zhang, Q. \& Zhao, Y., Finite-time consensus for a stochastic multi-species system

Wang, L.-P., see Zhou, L.-Y.

Wang, R. B., see Qu, J. Y.

Wang, W., see Zhang, J.

Wang, X.-N., see Zhou, L.-Y.

Wei, J., see Sun, X.

Wu, Q. \& Chen, S., Threshold analysis for epidemic models with high-risk immunization on networks

Xiang, H. L., see Tan, R. H.

Xie, D., Zhang, X., Yan, S. \& Gao, S., Permanence and extinction for a nonautonomous schistosomiasis model with saturation incidence rate

Xu, S. \& Bai, M., Time delays in proliferation process for solid avascular tumor under the action of external inhibitors

Yadav, A., Srivastava, P. K. \& Kumar, A., Mathematical model

3 (2015) 1550035

5 (2015) 1550063

5 (2015) 1550070

5 (2015) 1550068

2 (2015) 1550021

2 (2015) 1550018 for smoking: Effect of determination and education

1 (2015) 1550001

1 (2015) 1550008

2 (2015) 1550021

Yadi, K., see Borsali, F.

Yan, S., see Xie, D.

Yan, X.-P., see Zhang, C.-H.

4 (2015) 1550051

Yang, L., see Tian, B.

Yang, Y., Zhang, C. \& Jiang, X., Global stability of an SEIQV epidemic model with general incidence rate

Yao, Z., Almost periodic solution of Nicholson's blowflies model with linear harvesting term and impulsive effects

Yasmin, H., see Hayat, $\mathrm{T}$.

4 (2015) 1550044

2 (2015) 1550020

3 (2015) 1550053

2 (2015) 1550024

Yasmin, H., see Hayat, $\mathrm{T}$.

6 (2015) 1550073

1 (2015) 1550009

2 (2015) 1550020

Zhang, C., see Yang, Y.

Zhang, C.-H. \& Yan, X.-P., Formation of time patterns in a diffusive plantherbivore system with toxin-determined functional response

Zhang, J. \& Sun, J. Analysis of epidemic spreading with feedback mechanism in weighted networks

1 (2015) 1550007

Zhang, J., Zhang, Z. \&

Wang, W., Testing against second-order stochastic dominance of multiple distributions

3 (2015) 1550040

Zhang, P., see Ou Yang, H.-B.

3 (2015) 1550037

2 (2015) 1550025

Zhang, Q., see Wang, L.
Zhang, Q., see Zhang, X Zhang, X. \& Zhang, Q., Hopf analysis of $a$ differential-algebraic predator-prey model with Allee effect and time delay

3 (2015) 1550041

2 (2015) 1550021

6 (2015) 1550071

Zhang, Y., see Zhou, L.

Zhang, Z., see Zhang, J.
3 (2015) 1550040 
Zhao, Y., see Wang, L.

Zheng, J., see Wang, L.

Zhong, S., see Tian, B.

Zhou, L.-Y., Wang, X.-N., Wang, L.-P., Chen, Y.-Z. \& Jiang, X.-C., Genetic diversity of oil-tea camellia germplasms
2 (2015) 1550025

5 (2015) 1550063

4 (2015) 1550044 revealed by ISSR

analysis

5 (2015) 1550070

Zhou, L. \& Zhang, Y., Global exponential stability of cellular neural networks with multi-proportional delays

6 (2015) 1550071

Zhu, P., see Tan, C.

1 (2015) 1550004 